\title{
Development of a generalized Kitagawa diagram for cast G21Mn5 steel
}

\author{
Manuel Schuscha ${ }^{*}$, Martin Leitner, and Michael Stoschka \\ Christian Doppler Laboratory for Manufacturing Process based Component Design, Montanuniversität Leoben, Chair of Mechanical \\ Engineering, Leoben, Austria
}

\begin{abstract}
An accurate assessment of cast steel components including cast imperfections minimizes production rejects and moreover facilitates lightweight design. In order to improve the fatigue assessment of cast steel structures exhibiting macroscopic imperfections, a generalized Kitagawa diagram based on the linear elastic fracture mechanical theory is presented. Therefore, single-edge-notch-bending crack propagation tests as well as extensive fatigue tests utilizing un-notched base material specimens are conducted. Both the stress intensity factor threshold range and the plain material fatigue limit act as basis to set-up the generalized Kitagawa diagram. In addition, fatigue tests under rotating bending as well as axial loading using round V-notched specimens with varying opening angles are additionally conducted for validation. It was observed that the notch-stress-intensity-factor (NSIF) calculation based on El-Haddad's material length leads to slightly non-conservative designs for this cast material. But if the intrinsic material length is calculated by Neuber's stress averaging approach or Peterson's microstructural length instead, the presented NSIF-based fatigue assessment reveals a sound comparability to the experimentally determined fatigue reference strength. Summing up, the presented generalized Kitagawa diagram provides a feasible engineering-applicable fatigue assessment tool incorporating varying imperfection opening angles and load conditions for G21Mn5 cast steel.
\end{abstract}

\section{Introduction}

The aspect of a lightweight as well as economical design affects the dimensioning of modern high-performance components. To overcome this issue, it is required to enhance manufacturing process dependent fatigue assessment tools for the deployed materials. Despite quite high standards in casting simulation and manufacturing process technologies, the occurrence of imperfections, such as shrinkage-based porosities as well as sand, slag, or non-metallic inclusion, is unavoidable in cast steel.

Several fatigue approaches like Murakami's Varea concept [1] are suggested to properly assess such local imperfections, whereby recent studies [2, 3, 4] showed sound accordance with experimentally evaluated fatigue data. It should be noted that thereby, the fatigue dominating imperfection is characterized by the projected cross-section of the crack initiating defect, which is limited to comparably smaller defects with $\sqrt{ }$ area $<1000 \mu \mathrm{m}$. Supplementary to such stress-based methods, the linear elastic fracture mechanics (LEFM) considers the local stress field around the crack tip. In case of cast steel components, shrinkage porosities tend to reach larger dimensions due to the higher contraction during cooling, which corresponds to the multiple of the material intrinsic length, generally known as the ElHaddad length [5]. Due to this significantly enhanced dimensions of the imperfections, the application of Murakami's Varea concept is basically not valid anymore. Considering the size and spatial arbitrary shape of pores, one can treat flaws as cracks or internal notches, which enables the assessment of crack-like defects by LEFM and notch-like defects in a uniform way utilizing linear elastic notch fracture mechanics (LENFM).

Early studies [6, 7] observed that a linear elastic stress field in front of notch tip can be expressed in terms of plane problems, the so-called notch-stress-intensityfactor (NSIF), labelled as $\mathrm{K}_{\mathrm{I}}^{\mathrm{V}}$ for mode I and $\mathrm{K}_{\mathrm{II}}^{\mathrm{V}}$ for mode II: They generalize the stress intensity factor of a sharp crack toward the stress singularity at the notch tip for varying opening angles.

Considering a sharp V-notched component under tension mode I loading, the definition of the notch stress intensity factor $\mathrm{K}_{\mathrm{I}}^{\mathrm{V}}$ corresponding to Gross and Mendelson [7] equals to:

$$
\mathrm{K}_{\mathrm{I}}^{\mathrm{V}}=\sqrt{2 \pi} \lim _{\mathrm{r} \rightarrow 0}\left[\sigma_{\theta \theta}(\mathrm{r}, \theta) \mathrm{r}^{1-\lambda_{1}}\right]
$$

Herein, $\mathrm{r}$ and $\theta$ equal the polar coordinates around the notch tip, and $\lambda_{\mathrm{I}}$ is the Eigenvalue for mode I loading [6]. Furthermore, one can express the mode I NSIF $\mathrm{K}_{\mathrm{I}}^{\mathrm{V}}$ more generalized as follows: 


$$
\mathrm{K}_{\mathrm{I}}^{\mathrm{V}}=\alpha_{\gamma} \sqrt{\pi} \mathrm{a}^{\gamma} \sigma_{\mathrm{g}}=\sqrt{\pi}\left(\alpha_{\gamma}^{1 / \gamma} \mathrm{a}\right)^{\gamma} \sigma_{\mathrm{g}}
$$

Herein,

- $\quad \alpha_{\gamma}$ is a geometry shape factor, which depends on the overall geometry of the component, the opening angle, and the loading type

- $\gamma=1-\lambda_{\mathrm{I}}$ is the degree of the stress singularity for mode I loading

- $\quad a$ is the characteristic dimension, i.e. the notch or crack depth.

Originating from the applicability on sharp notches or reentrant corners, Atzori et al. [8] extended the Kitagawa diagram to blunt notches with a notch root radius $\rho$ and established a bridging between the defect sensitivity, referred to LENFM, and the notch sensitivity correlated to linear notch mechanics. Several studies [9-11] applied this approach mainly focusing on carbon steels and aluminum alloys. A sound conformity between the evaluated fatigue test results and the NSIF-concept regarding different notch acuities was achieved.

For sharply notched components, the fatigue strength is affected by their stress singularity exponent $\gamma$. Evaluating parts with varying opening angles $\varphi$ leads to different stress singularities and therefore disables the possibility to compare the NSIF's directly as fatigue strength qualifier. Former studies [12, 13] proposed an advanced energy-based approach to estimate static and cyclic behavior of cracks and notches.

\section{Numerical Analysis}

Due to the spatial arbitrary shape of porosities, which are found in steel castings, it is intended to cover the fatigue assessment of different types of macroscopic defects in a uniform way. Therefore, three round specimens with circumferential notches were defined representing cast steel imperfections with varying opening angles. To correlate the fatigue behavior of crack-like defects as well as more globular shrinkage porosity, the following opening angles $\varphi$ were defined: $45^{\circ}, 90^{\circ}$ and $135^{\circ}$. To obtain fatigue test results similar to steel castings with macroscopic imperfections, the notch depth was consistently set to $3 \mathrm{~mm}$. It should be noted that the fatigue effective notch depth $\mathrm{a}_{\mathrm{eff}}$ increases based on the overall specimen geometry and hence, additionally the applicable shape factor $\alpha_{\gamma}$ enhances as exemplified in Eq.(3). To facilitate the utilization of the NSIF concept, the notch root radius was set to $\rho=0.1 \mathrm{~mm}$ to ensure the condition $\rho<<$ a.

Numerical analyses of the aforementioned three specimen geometries were conducted as linear-elastic studies utilizing the software package Abaqus to evaluate the associated NSIF's. The material and simulation parameters, which were used to set-up the numerical model, are listed in Table 1.

Table 1. Material and simulation parameters

\begin{tabular}{|c|c|}
\hline Parameter & Value \\
\hline
\end{tabular}

\begin{tabular}{|l|c|}
\hline Young's Modulus [GPa] & 210 \\
\hline Poisson's ratio [-] & 0.3 \\
\hline Element type & CAX8 / CAXA84 \\
\hline
\end{tabular}

At this point it should be pointed out that all stress values within this paper, except the plain fatigue limits and the notch stresses, are referred to the gross section of the specimens. Referencing to the gross stress enables a fatigue assessment without defining an actual net crosssection. This procedure is feasible especially in case of complexly-shaped components, at which a net crosssection is generally not simply definable. For example in case of a shrinkage porosity-afflicted component under axial loading a definition of the size of the supporting cross-section may not be simple. The reference to the gross section and an accordingly defined imperfection based geometry factor allows a more engineeringfeasible straightforward assessment of local fatigue strength.

The numerical analyses of the specimen geometries were done as linear-elastic simulation runs with unified nominal gross stress amplitude of one Mega-Pascal. The notches were sketched with a notch root radius of zero to obtain the stress distribution and its singularity quite close to the notch root, which is shown in Fig.1. Due to the double logarithmic scale, the slope of the resulting curve represents the degree of singularity $\gamma$ given by the specific notch opening angle. In case of the $45^{\circ}$ notched specimen, the slope equals to 0.495 . This is similar to the well-known singularity of a crack, which possesses a value of one-half. The calculation of the notch stress intensity factor $\mathrm{K}_{\mathrm{I}}^{\mathrm{V}}$ was conducted on the basis of Eq.(1). Hence, the related NSIF keeps constant over the radial distance $r$ from the notch tip.

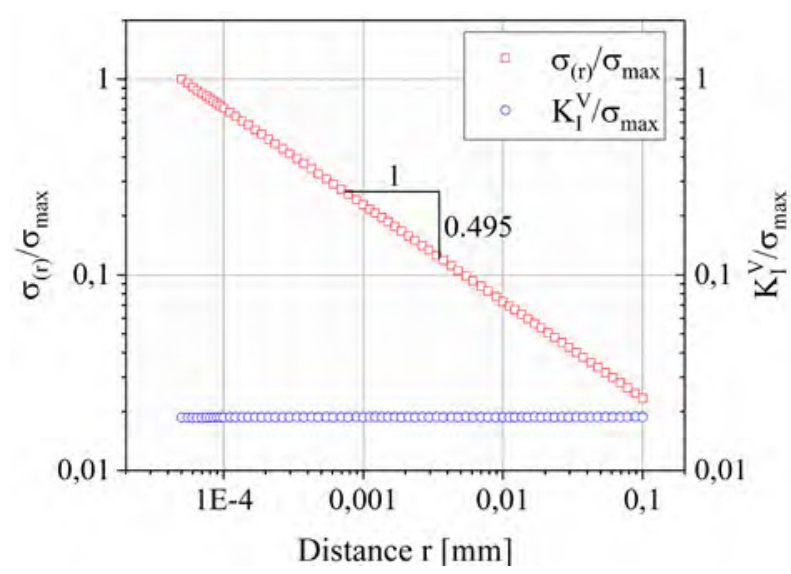

Fig.1 Normalized Stress and KIV relative to the notch root

Furthermore, the mode I notch stress intensity factors for the remaining opening angles under bending as well as for axial loading were calculated in the same manner. The resulting NSIF factors are listed in Table 2.

Table 2. Mode I NSIF's for bending and axial loading related to a unified nominal gross stress

\begin{tabular}{|c|c|c|}
\hline \multirow{2}{*}{ Opening Angle } & \multicolumn{2}{|c|}{ NSIF $\mathbf{K}_{\mathbf{I}}^{\mathbf{V}} / \sigma_{\mathrm{g}}\left[\mathbf{m m}^{\gamma}\right]$} \\
\cline { 2 - 3 } & Bending & Axial Loading \\
\hline $45^{\circ}$ & 6.02 & 4.80 \\
\hline
\end{tabular}




\begin{tabular}{|c|c|c|}
\hline $90^{\circ}$ & 6.61 & 5.22 \\
\hline $135^{\circ}$ & 7.80 & 5.89 \\
\hline
\end{tabular}

Moreover, the specimen's notch stress intensity factors can be utilized to calculate the effective notch depth $a_{\text {eff }}$ by means of Eq.(2), which can be understood as the equivalent size of a crack in an infinite plate, based on the derived NSIF value. In regard to the calculation of $a_{\text {eff }}$ for different opening angles $\varphi$, it is necessary to apply the corresponding degree of stress singularity $\gamma$ for each notch shape. In addition, the shape factor $\alpha_{\gamma}$, which depends on the overall sample geometry, the load case, and the notch shape, can be directly deduced from $\mathrm{a}_{\mathrm{eff}}$, as exemplified in Eq.(3).

$$
\mathrm{a}_{\mathrm{eff}}=\alpha_{\gamma}^{1 / \gamma} \mathrm{a}
$$

Finally, the corresponding effective notch sizes $a_{\text {eff }}$ are determined for each load case and opening angle based on the acting NSIF-values. The results are summarized in Table 3.

Table 3. Mode I NSIF's for bending and axial loading related to a uniform nominal gross stress

\begin{tabular}{|c|c|c|}
\hline \multirow{2}{*}{ Opening Angle } & \multicolumn{2}{|c|}{$\mathbf{a}_{\text {eff }}[\mathbf{m m}]$} \\
\cline { 2 - 3 } & Bending & Axial Loading \\
\hline $45^{\circ}$ & 11.82 & 7.48 \\
\hline $90^{\circ}$ & 17.95 & 10.68 \\
\hline $135^{\circ}$ & 94.18 & 39.79 \\
\hline
\end{tabular}

The obtained values of $a_{\text {eff }}$ significantly increase with the notch opening angle. Hence, both the notch depth as well the notch opening angle affect the endurable fatigue strength.

Based on these results, the fatigue strength for the three samples with varying notch acuity can be assessed in terms of the generalized Kitagawa diagram. The location on the abscissa is defined by the effective notch depth of each specimen geometry. In order to estimate the fatigue limit of the specimens, experimental crack growth tests ought to supply the materials threshold value, which can be further used to determine the intrinsic El-Haddad value for a specific load ratio. The ratio of the NSIF values compared to the $\mathrm{K}_{\mathrm{I} \text {,th }}^{\mathrm{V}}$ supports a proper estimation of the specimen's fatigue limit.

Concluding the numerical analyses, the following procedure has to be maintained to obtain proper NSIFvalues for fatigue assessment purposes.

- A very fine mesh, down to $10^{-4}-10^{-5} \mathrm{~mm}$, should be utilized to properly consider the notch stress singularity.

- To obtain the NSIF value, the notch has to be modelled without a notch tip radius.

- The evaluation of the NSIF uses the principal stress component, which is in agreement with the axial specimen axis.

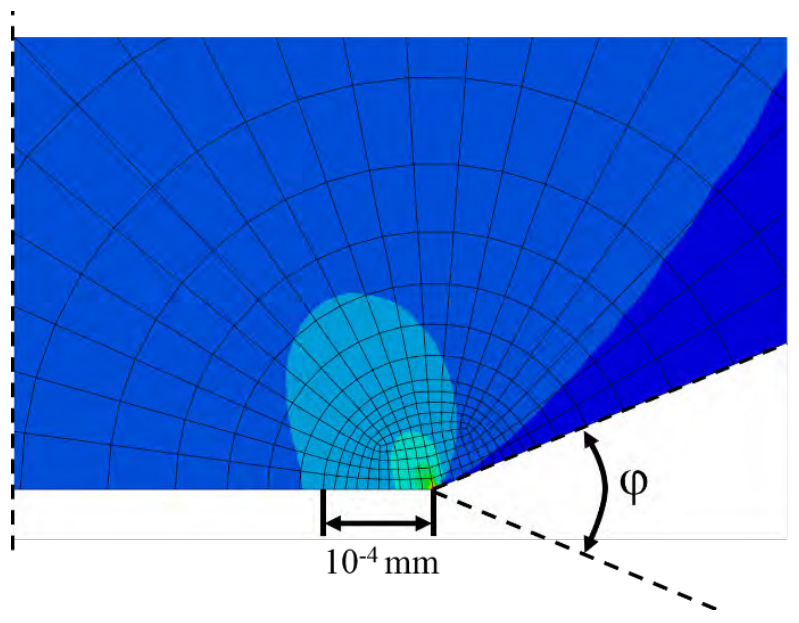

Fig.2 Mesh size at the notch root of the axisymmetric model

\section{Development of the generalized Kitagawa Diagram}

In contrast to the Kitagawa-Takahashi diagram for crack-like defects, the generalized form enables the application for open notches. The generalized Kitagawa diagram (GKD) can be divided in three basic sections, which feature different fatigue approaches dependent on the effective imperfection size.

The first section, located on the left side of the diagram below the intrinsic material length $\mathrm{a}_{0}[5,14]$, generally known as the El-Haddad length, can be defined as the short crack region. In case of a GKD, the intrinsic material length of V-notches is defined similarly to ElHaddad-Smith-Topper length parameter as the intersection of $\Delta \sigma_{\mathrm{g}, \mathrm{th}}$ and $\Delta \sigma_{0}[15]$.

$$
\mathrm{a}_{0}^{\mathrm{v}}=\left(\frac{1}{\sqrt{\pi}} \frac{\Delta \mathrm{K}_{\mathrm{l}, \mathrm{th}}^{\mathrm{v}}}{\Delta \sigma_{0}}\right)^{1 / \gamma}
$$

A defect of this length has supposedly no characteristic impact on the component's fatigue resistance. Therefore, the short defect region, strictly assuming no further short crack growth during cyclic loading, exhibits an endurance limit as characteristic fatigue strength. If the effective defect increases, the GKD is controlled by the LENFM and is therefore represented by a straight line in a double-logarithmic scale. The area on the right hand side of the diagram illustrates the third section, which is dominated by the linear elastic notch mechanics in case of extremely huge imperfections. If a defect size is greater than the intersection point $\mathrm{a}^{*}$, the LENFM is no longer applicable to assess the fatigue strength properly. This intersection point is defined by the geometric correlation:

$$
\mathrm{a}^{*}=\mathrm{K}_{\mathrm{t}}^{2} \cdot \mathrm{a}_{0}^{\mathrm{V}}
$$


Figure 3 provides an overview about the three basic regions of the generalized Kitagawa diagram.

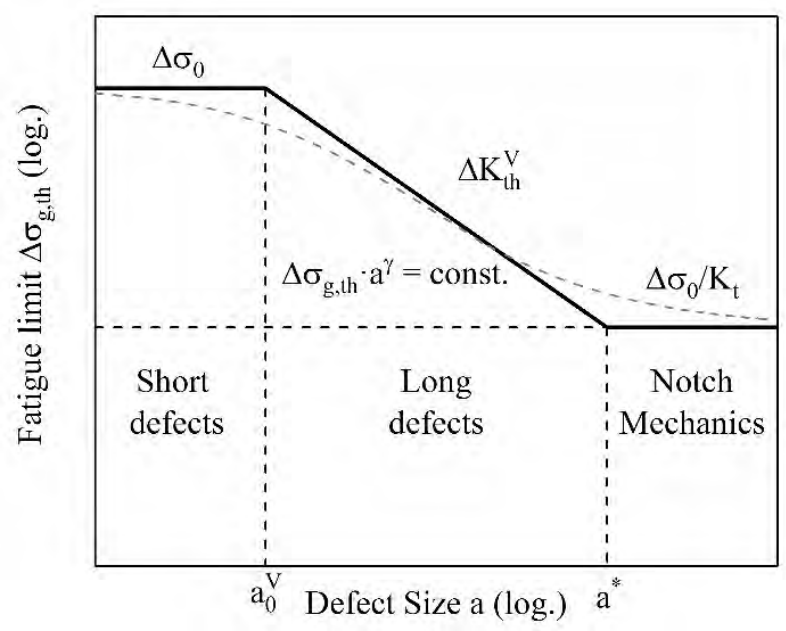

Fig.3 Structure of the generalized Kitagawa diagram (GKD)

Several parameters, e.g. notch opening angle, stress concentration factor and load ratio, affect the shape of the GKD. The material's plain fatigue limit influences the short defect region in case of $\Delta \sigma_{0}$ and the intrinsic material length as stated in Eq.(4). Based on the opening angle $\varphi$ and Williams' equation, the Eigenvalue $\lambda$ and the degree of singularity $\gamma$ can be determined, which affect both the position of the intersection point on the left side as well as the slope of the LENFM section. An increase of the opening angle $\varphi$ leads to a lowered stress singularity as well as to larger values of $\mathrm{a}_{0} \mathrm{~V}$. Furthermore, the slope of the LENFM region decreases with rising $\varphi$. In the notch mechanics applicable design region on the right hand side of the diagram, it is worth mentioning that the stress concentration factor $\mathrm{K}_{\mathrm{t}}$ affects the position of the transition point $\mathrm{a}^{*}$. A comparably high notch acuity leads to a high stress concentration factor and therefore, the value of $\mathrm{a}^{*}$ is shifted towards larger defect sizes.

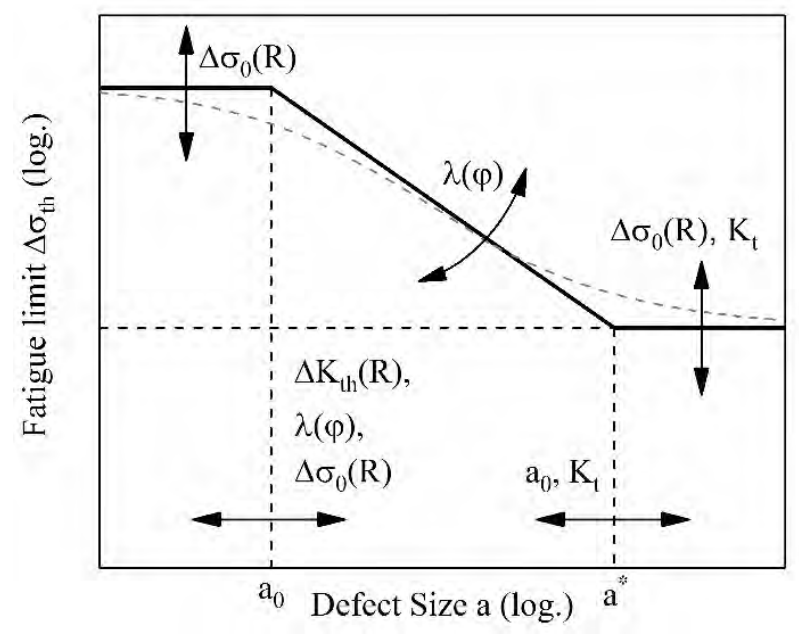

Fig.4 Influencing parameters on the GKD
To draw the generalized Kitagawa diagram, two base material parameters need to be determined first, namely the plain fatigue limit $\Delta \sigma_{0}$ and the threshold stress intensity factor range $\Delta \mathrm{K}_{\text {th }}$. The plain fatigue resistance can be obtained by testing un-notched specimens under different load cases and load ratios. Concerning the determination of the long crack threshold, the crack growth behavior of single-edge notched bending (SENB) specimens can be assessed for same load ratios as in case of cyclic testing un-notched specimens. Based on these two material parameters, the El-Haddad's intrinsic material length $\mathrm{a}_{0}$ can be estimated.

\section{Fatigue testing}

The alternating cyclic tests were performed on a rotating bending test rig as well as on an axial fatigue test machine. The corresponding load stress ratio is set to a value of $R=-1$. The abort criterion of the fatigue tests is total burst failure in the finite life region or defined as a run-out if ten million load cycles are reached. It should be noted that no endurance limit is feasible within this limited load cycle range [16]. The assessment of the test results was conducted by the ASTM E739 [17] and the $\arcsin \sqrt{ } \mathrm{P}[18]$ approach for the finite-life and high-cycle fatigue region, respectively. 

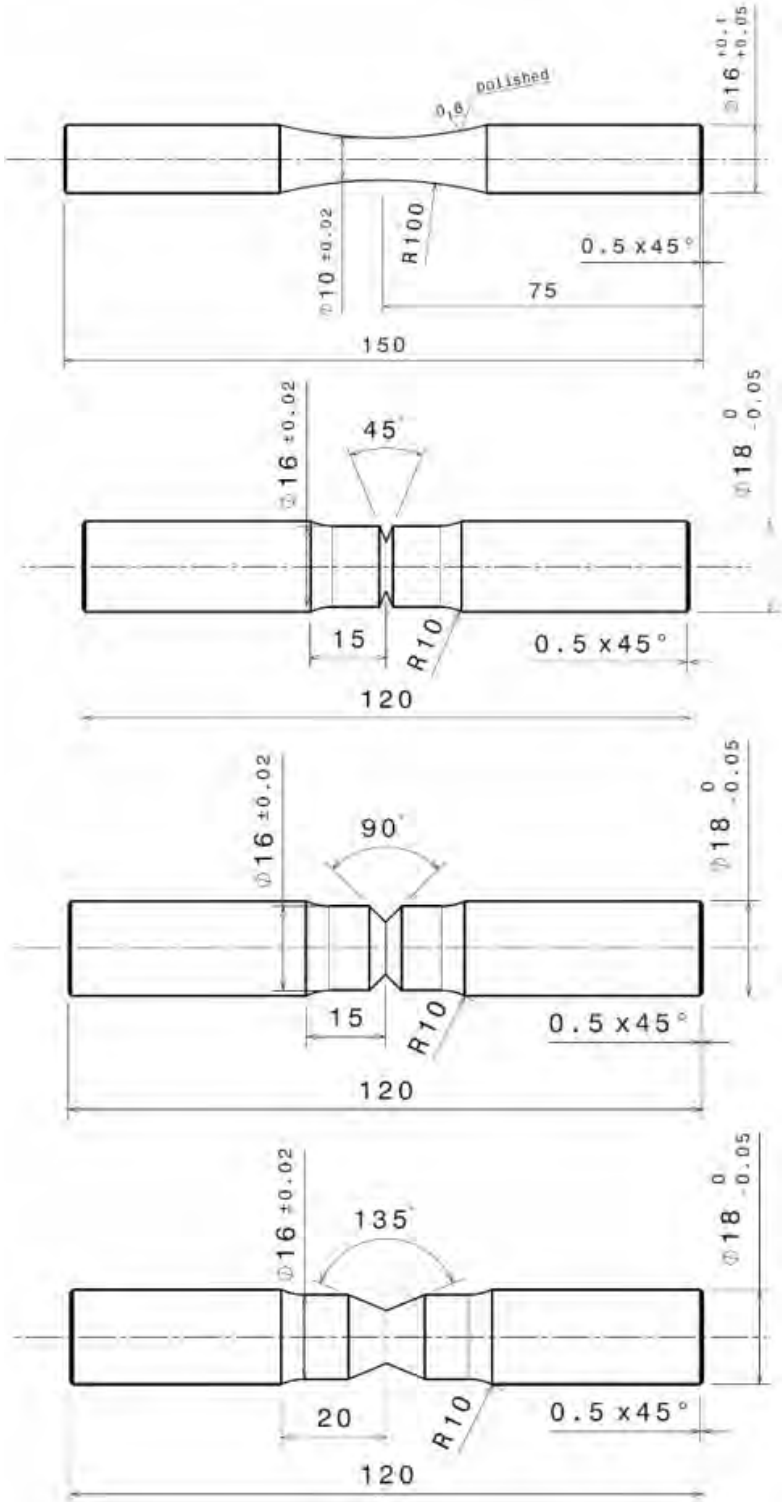

Fig.5 Geometries of un-notched and notched fatigue specimens

Concerning the tested fatigue specimens, Fig.5 presents the un-notched and V-notched geometries. The notched specimen series possess a sharp notch with a tip radius smaller than ten microns. The un-notched, plain specimen geometry features a nominal testing diameter of $10 \mathrm{~mm}$. The notched specimens exhibit $16 \mathrm{~mm}$ for the gross and $10 \mathrm{~mm}$ for the net section diameter. The investigated material is a cast steel G21Mn5, equivalent to grade ASTM 352, in normalized condition.

Table 4. Chemical composition of the evaluated cast steel G21Mn5 in weight percent

\begin{tabular}{|c|c|c|c|c|}
\hline $\mathrm{C}$ & $\mathrm{Si}$ & $\mathrm{Mn}$ & $\mathrm{P}$ & $\mathrm{S}$ \\
\hline $0.21 \%$ & $0.47 \%$ & $1.1 \%$ & $0.01 \%$ & $0.004 \%$ \\
\hline
\end{tabular}

In addition, the microstructure of the heat-treated cast steel is investigated. A three per cent alcoholic nitric acid is used as etchant.

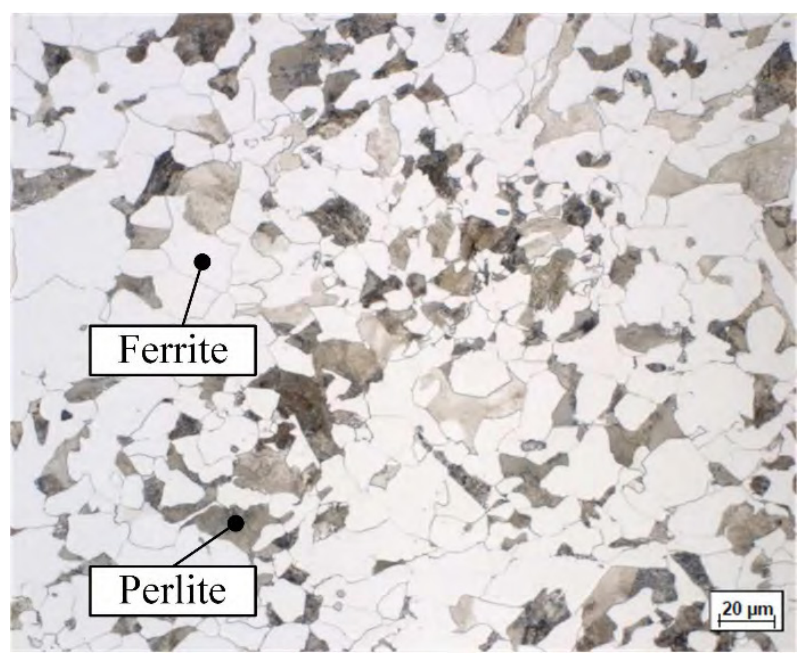

Fig.6 Microstructure of normalized G21Mn5

The perlitic islands in Fig.6 occur quite uniformly distributed within the ferritic matrix in metallographic sections taken out of fatigue test samples.

Regarding the rotating bending fatigue test results, the evaluated $\mathrm{S} / \mathrm{N}$-curves reveal an increase in fatigue limit with an enhanced notch opening angle. This behavior is expected as an increasing notch stress intensity factor reduces the nominal gross section based fatigue strength. The statistically evaluated high-cycle fatigue strength values of the $45^{\circ}$ and $90^{\circ}$ notched Vspecimen exhibit only a fraction of about $75 \%$ to $80 \%$ compared to the $135^{\circ}$ notched samples. Furthermore it can be stated that a rising stress concentration factor leads to a narrower scatter band of the results as well as an increase of the slopes in the finite-life region. As the investigated material is a cast steel thus featuring imperfections such as shrinkage based micro-pores in the base material, the second slope of the $\mathrm{S} / \mathrm{N}$ curves in the high-cycle fatigue region was set to a decrease of ten percent stress level per decade load cycles [19]. Moreover, a direct comparison of the results indicates that the fatigue strength relation between the different specimens is non-linear, despite an equidistant net section. The difference among the $45^{\circ}$ and $90^{\circ}$ geometries is smaller than those two compared to the $135^{\circ}$ samples. This tendency is valid both for the fatigue limits as well as the slope and the scatter band values. It deduces that the notch opening angle has a severe effect on the gross section based fatigue strength. Fig.7 depicts an overview of the test results of the rotating bending test series for all three specimen types. All fatigue test values are normalized to the statistically evaluated highcycle fatigue strength at ten million load-cycles for the $135^{\circ}$ specimen at a survival probability of $50 \%$. 


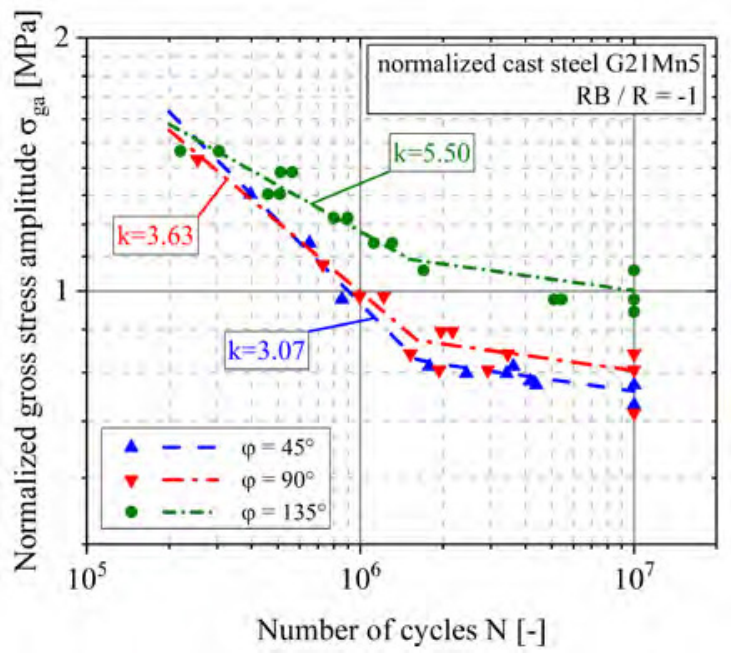

Fig.7 S/N curves of the rotating bending test

Subsequently, light optical microscopy (LOM) and scanning electron microscopy (SEM) were conducted on specimens under bending as well as under axial loading to analyze the location crack initiation. Fracture surface analysis of the V-notched specimens showed an equally distributed circumferential crack initiation for the entire test series. Fig. 8 and Fig.9 illustrate the fracture surfaces of two rotating bending samples with $90^{\circ}$ opening angle, tested at different load-levels.

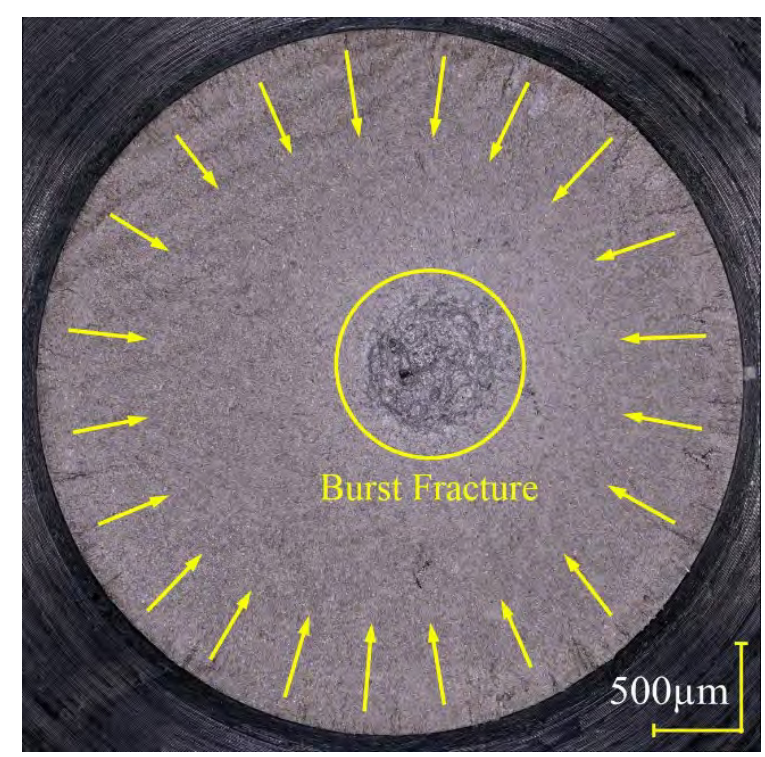

Fig.8 Rotating bending specimens: $\sigma_{\text {norm }}=0.81 ; \mathrm{N}=3 \cdot 10^{6}$

Due to the quite high notch acuity both specimen show similar behavior regarding crack initiation. Several microscopic crack initiation planes can be found by scanning electron microscopy (SEM), which coalesce during further cycling loading. In addition, some minor defects are recognizable on the fracture surfaces. Despite the occurrence of such local shrinkage porosities, the rotating bending and axial loading test results show no significant influence of these small defects on the highcycle fatigue endurance limit of notched specimen.

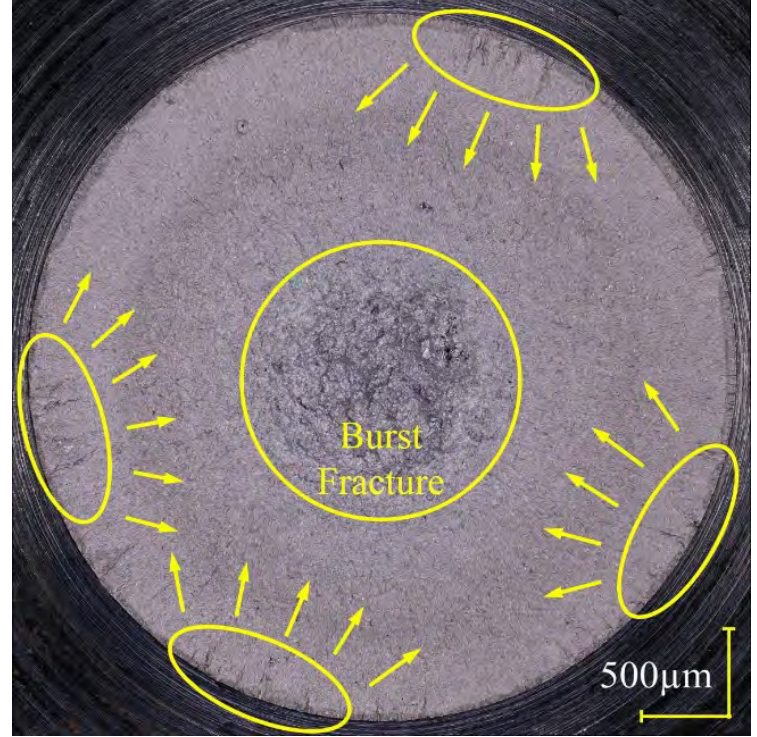

Fig.9 Rotating bending specimen: $\sigma_{\text {norm }}=1.43 ; \mathrm{N}=2.5 \cdot 10^{5}$

A scanning-electron-microscopy image of a small defect found at an un-notched rotating bending specimen is shown in Fig.10. However, it is recognizable by fracture surface analysis that these pores do not majorly affect the short crack propagation phase and hence, no significant impact on the lifetime is given.

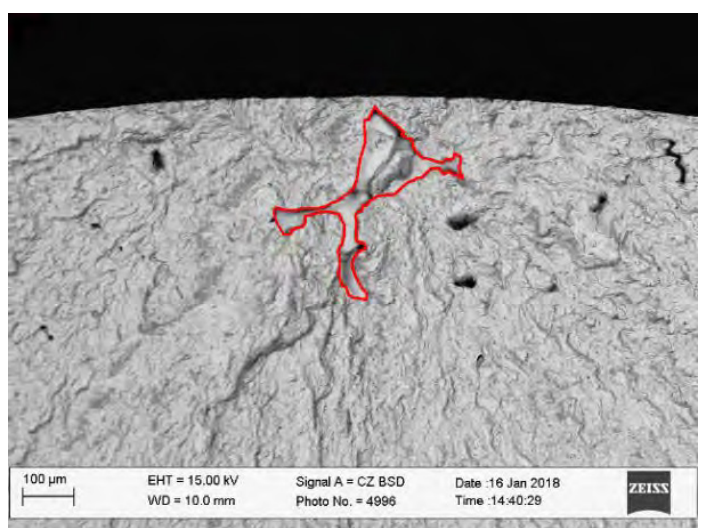

Fig.10 SEM image of an defect (un-notched RB): $\sigma_{\text {norm }}=1.08$; $\mathrm{N}=1.9 \cdot 10^{6}$

On the contrary to notched specimen, the test results as well as the fracture surfaces of the un-notched, axial loaded specimens exhibit a pronounced effect on bulk fatigue strength by shrinkage porosity. The uniform stress distribution under axial loading in case of unnotched specimens features the crack growth of defects. For example, the specimen displayed in Fig.11 failed at $\mathrm{N}=1.7 \cdot 10^{6}$ load cycles even though the load level is below the evaluated endurance limit of the cast steel. As the goal of the un-notched test series is to determine the plain high-cycle fatigue strength, test results with such huge casting imperfections are excluded from the statistical evaluation. 


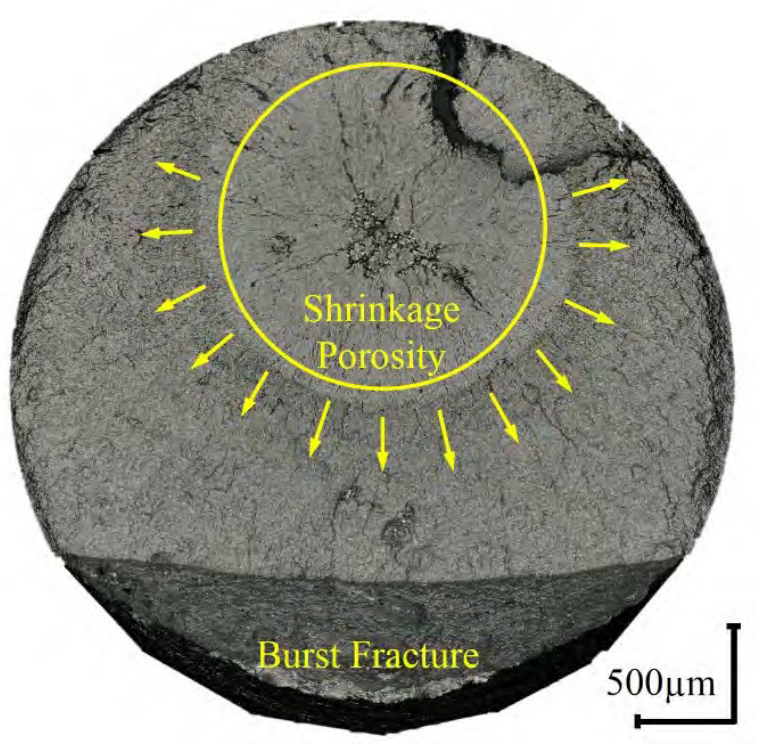

Fig.11 Critical defect within un-notched specimens

Summing up, the occurrence of shrinkage porosity is unavoidable in cast materials, especially in cast steel due to the high casting temperature and subsequent contraction of the material during cool-down. As long as the equivalent square defect length is below one millimeter, the application of Murakami's Varea approach can be easily utilized, but for larger imperfections or even networks of shrinkage porosity the NSIF-values may be used to gain insight into the local fatigue strength of arbitrary shaped defects.

\section{Discussion}

The assessment of the fatigue tests including plain and $\mathrm{V}$-notched specimens as well as the crack growth tests were utilized to set-up the generalized Kitagawa diagram for the investigated G21Mn5 cast steel. It was found that the calculation of the material's intrinsic length $\mathrm{a}_{0}$ based on the El-Haddad-Smith-Topper equation leads to a value of about one-hundred and eighty microns. This would lead to a non-conservative deviation of the NSIFcalculation based fatigue limits compared to the experimentally obtained endurance fatigue strength at ten million load cycles. This discrepancy persists approximately constant for all three evaluated notch geometries and both load cases and ranges up to about thirty percent in fatigue strength. It should be mentioned that this deviation can be traced back to a change in stress intensity threshold value of about one to a squareroot of two.

Due to the alternating testing implying a stress ratio of $\mathrm{R}=-1$, crack closure effects may occur during the crack initiation and crack propagation phase [20]. This effects builds up during the growth of the crack; hence, the effect enhances from short to long crack growth [21]. Therefore, the effective threshold stress intensity factor for short cracks is lower than the threshold value for long cracks. This behavior is known as the crack resistance (R)-curve. For the determination of the effective SIF, the $45^{\circ}$ results were taken to recalculate the long crack threshold value and further on the El-Haddad length for sharp cracks is very close to the singularity of the Vnotch. Therefore, the V-notch can be treated as a crack of the same length [22]. The results show an accurate, even slightly conservative estimation of the NSIF approach, which is illustrated in Fig.12. It should be mentioned that this recalculated intrinsic length matches exactly if El-Haddad's length is divided by a factor of two.

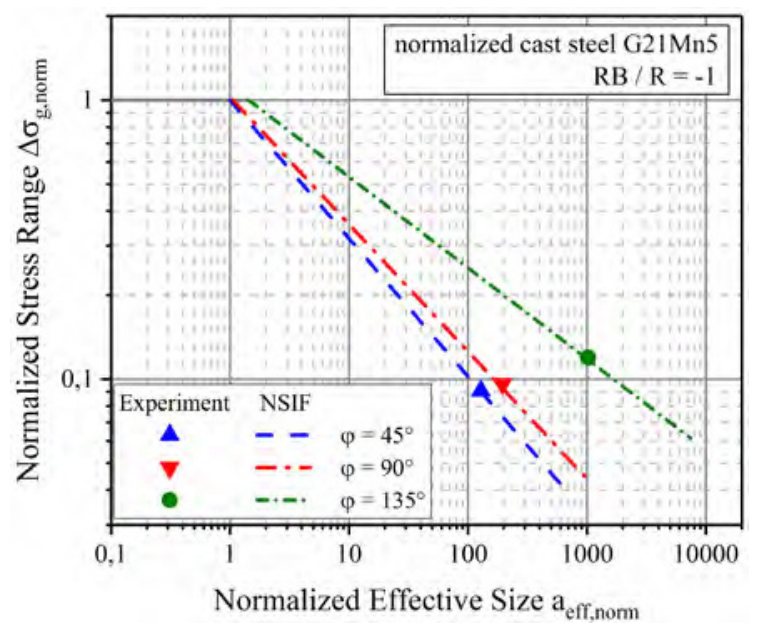

Fig.12 Generalized Kitagawa diagram in comparison with RB fatigue test results

A comparison of the NSIF-based calculated and experimentally evaluated fatigue strength values up to ten million load cycles reveals a sound agreement with a maximum scatter of only three percent.

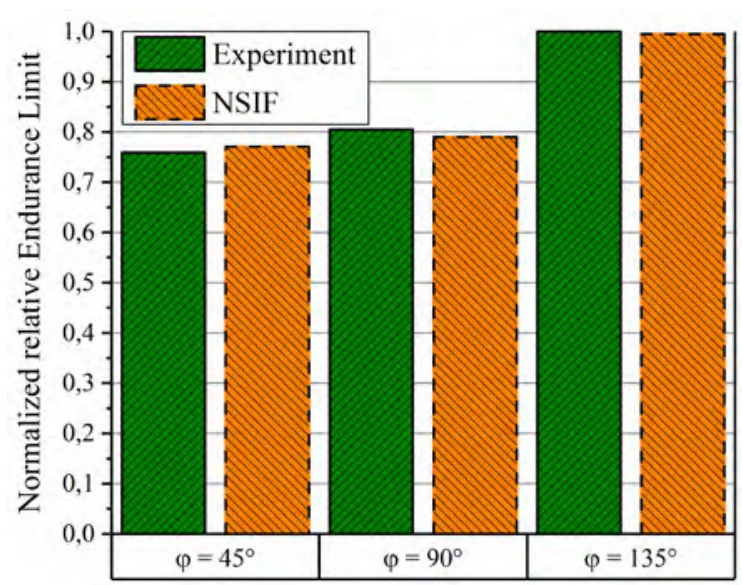

Fig.13 Normalized estimated vs. experimental results

Finally, it should be noted the critical distance theory [14] provides an alternative fatigue test sample based approach to determine the microstructural support length if $\mathrm{S} / \mathrm{N}$-curves with different notch factors are available. Applicable methods are the point and the line method which are both applied for the un-notched and notched fatigue results. Both approaches lead to a reduction of the proposed intrinsic length value to about ninety microns. This leads also to a sound match between the experimental fatigue results and the 
proposed mean fatigue limit deduced by the generalized Kitagawa-diagram.

\section{Summary and Conclusions}

Firstly, a numerical simulation of V-notched specimens with opening angles of $45^{\circ}, 90^{\circ}$ and $135^{\circ}$ under bending and axial loading was conducted. Further on, the essential values for the application of the linear elastic notch fracture mechanics in terms of the notch stress intensity factor and an effective notch depth, were calculated. Fatigue test results under rotating bending and axial loading illustrate primarily a relation between the notch acuity, the opening angle and the resulting $\mathrm{S} / \mathrm{N}$-curve slope as well as the individual fatigue limits.

Secondly, the NSIF approach was utilized to calculate the essential values of the generalized Kitagawa-diagram for varying specimen geometries and notch acuities. It enables the study of notch designs on gross fatigue strength in a unified manner.

Thereby, the calculated values for G21Mn5 cast steel showed a non-conservative deviation of up to thirty percent compared to validating fatigue test results if ElHaddad's material length is used. This intrinsic material length leads to about one-hundred and eighty microns in case of calculation with the long crack growth threshold.

To overcome this discrepancy, Taylor's theory of critical distance, applied both for line- and point method, lead to a value of exactly half of El-Haddad's length. This heads to a reduced value of ninety microns as microstructural support length for G21Mn5, which matches the experimental data well. Since the degree of stress singularity of a crack and the V-notch with an opening angle of $45^{\circ}$ are very close, a final recalculation of the stress intensity factor based on the notched specimen is supported. The results show a sound accordance of the estimated and experimentally determined fatigue limits for varying notch acuities. They prove the basic applicability of the NSIF-concept both for rotating bending and axial loading as fatigue design strength parameter for G21Mn5 cast steel.

Further work will focus on the effect of crack closure effects on the effective stress intensity factor range in terms of R-curves and especially its subsequent impact on generalized Kitagawa diagram based fatigue strength.

Summing up, the presented approach facilitating the generalized Kitagawa diagram for cast steel G21Mn5 demonstrates an unrestricted applicability of the NSIF concept and will be further investigated for porosity afflicted cast steel components.

The financial support by the Austrian Federal Ministry for Digital and Economic Affairs and the National Foundation for Research, Technology and Development is gratefully acknowledged.

\section{References}

1. Y. Murakami, Metal Fatigue: Effects of Small Defects and Nonmetallic Inclusions (2002)
2. Y. Murakami, Int. J. Fatigue, 41, 2-10 (2012)

3. Y. Yamashita, Y. Murakami, Int. J. Fatigue, 93, 406-414 (2016)

4. D. Krewerth, T.Lippmann, A. Weidner, H. Biermann, Int. J. Fatigue, 84, 40-52 (2016)

5. M.H. El Haddad, T.H. Topper, K.N. Smith, Eng. Fract. Mech., 11, 573-584 (1979)

6. M. L.Williams, J. Appl. Mech., 19, 526-528 (1952)

7. B. Gross, A. Mendelson, Int. J. Fract., 8, 267-276 (1972)

8. B. Atzori, P. Lazzarin, Int. J. Fatigue, 107, 1-8 (2001)

9. B. Atzori, P. Lazzarin, G. Meneghetti, Fatigue Fract. Eng. Mater. Struct., 26, 257-267 (2003)

10. B. Atzori, P. Lazzarin, Int. J. Fract., 118, 271-284 (2002)

11. P. Lazzarin, F. Berto, Int. J. Fract., 135, 33-38 (2005)

12. G. Glinka, Eng. Fract. Mech., 22, 485-508 (1985)

13. P. Lazzarin, R. Zambardi, Int. J. Fracture, 112, 275298 (2001)

14. D. Taylor, Int. J. Fatigue, 21, 413-420, (1999)

15. B. Atzori, P. Lazzarin, G. Meneghetti, Int. J. Fract., 133, 61-87 (2005)

16. B. Pyttel, D. Schwerdt, c. Berger, Int. J. Fatigue, 33, 49-58 (2011)

17. ASTM E739

18. D. Dengel, J. Materialwiss. Werkstofftech, 8, 253288 (1975)

19. C.M. Sonsino, Int. J. Fatigue, 29, 2246-2258 (2007)

20. R. Pippan, A. Hohenwarter, FFEMS, 40, 471-495 (2017)

21. B. Tabernig, R. Pippan, Eng. Fract. Mech., 69, 899907 (2002)

22. G. Meneghetti, S. Masaggia, World Foundry Congress, (2012) 